\title{
Pediatric musculoskeletal imaging: beyond the basics
}

\author{
J. Herman Kan • R. Paul Guillerman • Michael J. Callahan
}

Received: 13 November 2012 / Accepted: 15 November 2012

(C) Springer-Verlag Berlin Heidelberg 2012

Under the guidance of Drs. J. Herman Kan, R. Paul Guillerman and Michael J. Callahan, with the administrative assistance of Karen Schmitt, the SPR organized the second pediatric musculoskeletal meeting, "Pediatric Musculoskeletal Imaging: Beyond the Basics," in Key Biscayne, FL, on Sept. 21-23. The 2012 meeting featured 13 course faculty members, including ten pediatric radiologists, one adult musculoskeletal radiologist and two orthopedic surgeons, who collectively presented 34 lectures in addition to a hands-on demonstration of common orthopedic measurements. There were more than 200 attendees at the meeting, which was held at the Ritz-Carlton Key Biscayne. The conference presentations were divided into sections, including imaging of the lower extremity, imaging of the upper extremity, unique entities and controversies, and general topics in pediatric musculoskeletal imaging.

The meeting covered the full breadth of modalities employed in pediatric musculoskeletal imaging, including radiography, CT, MRI, US and interventional techniques, as well as a broad spectrum of pathologies including infectious, inflammatory, neoplastic, developmental and post- traumatic entities. The meeting focused particular attention on the many challenges of performing and interpreting imaging studies on children, where specialized knowledge of the growing and maturing musculoskeletal system is required and issues such as sedation and ionizing radiation exposure are more germane than in adults. The meeting provided an overview of existing and potential pediatric musculoskeletal applications of US imaging to increase awareness of this valuable modality that does not require sedation or incur ionizing radiation exposure in children. By including several lectures by orthopedic surgeons, the meeting also emphasized the importance of perspective and communication of clinically relevant information among specialists.

"Pediatric Musculoskeletal Imaging: Beyond the Basics" provided a convenient forum for attendees to learn in depth about current topics related to the practice of pediatric musculoskeletal imaging. This supplement to the Pediatric Radiology journal contains peer-reviewed manuscripts highlighting material presented at the conference, so that the knowledge imparted may more widely benefit the SPR membership and broader radiology community.
J. H. Kan $(\bowtie) \cdot$ R. P. Guillerman

E. B. Singleton Department of Pediatric Radiology,

Texas Children's Hospital, 6701 Fannin St., Suite 470,

Houston, TX 77030, USA

e-mail: jhkan@texaschildrens.org

M. J. Callahan

Department of Radiology, Children's Hospital Boston, Harvard Medical School, Boston, MA, USA 